\title{
Research on the Role of Translation Theory on Translation Teaching
}

\author{
Yu Fang \\ Changchun University of Chinese Medicine, Changchun, Jilin, 130117
}

Keywords: application research; statistical process control; quality management

\begin{abstract}
The relationship between translation theory and translation practice has been discussed in the translation field in China for many years. Everyone has a good understanding of this. However, some translation teachers are still unclear because they are responsible for a large number of translation teaching tasks. If there is a vague or even erroneous understanding of this issue, it will affect the content, teaching methods and research of translation teaching. Therefore, it is necessary to discuss the relationship between translation theory and translation practice and the position of translation theory in translation teaching.
\end{abstract}

\section{Introduction}

The traditional translation teaching is based on translation exercises, teaches translation skills, and seldom teaches translation theory; even if it teaches, it is nothing more than an empty interpretation of the meanings of faith, dharma, or faithfulness and fluency. It does not play a guiding role for practice. From the mid-1980s onwards, the study of domestic translation theories presented a prosperous scene, and various major theoretical schools abroad were continuously introduced to the Chinese translation community. People finally got rid of the shackles of empiricism and began to examine translation teaching from the perspective of theory. They realized that translation theory is not only a summary of the translator's experience in translation work, but can only be understood and conveyed, but can be from a scientific perspective. Summarize and find out the laws, which are used to guide one's knowledge of practice. The author has discovered from translation textbooks published in recent years that the content of translation theories has increased, such as Fang Mengzhi. Translation courses are usually composed of four parts: translation practice, basic knowledge, translation theory, and the history of translation thoughts. The translation theory should play a very important role in the overall quality education because the relationship between translation theory and translation teaching is very close. Yes, translation teaching cannot be without theoretical guidance. In fact, the combination of translation theory teaching and translation practice is a dialectical unity of science, which is consistent with and embodies the overall quality teaching concept. So, what percentage of translation theory should be used in translation teaching is a new topic. This article proposes to discuss this issue.

\section{Positioning and Characterization of Translation Theory}

Many famous scholars at home and abroad believe that translation theory and translation studies are the same concept. For example, Mr. Tan Zaixi wrote in his "Translation Studies”, “The specific research object of translation studies should include the essence of translation, the principles and standards of translation, the methods and techniques of translation, the operating procedures and procedures of translation, and the process of translation. The various contradictions that emerged....” Next, he wrote, “A complete set of translation theories includes: clarification of the essence of translation, describing the process of translation, determining the principles and standards of translation, describing the method of translation, and explaining the translation The various contradictions in the study." Professor Fan Shouyi also said, "What kind of theory should translation have? We don't think it is important to call him "translatology". 'There are also many areas in the field of translation studies. Classification methods. Prof. Yang Zixue translated the translation studies into three levels: translation engineering, translation art, and translation studies. 
Professor Fan Shouyi divided it into three parts: basic theory, application skills, and multi-view research. Professor Tan Zai-chi uses linguistics to divide his discipline into three parts: general translation, special translation, and applied translation. Professor Cao Minglun learns from the influence of literary studies on the subject category and divides the subject areas of translation studies into translation theory, translation history and translation criticism. According to Professor Cao Minglun's theory, this icon clearly illustrates the importance of translation theory, which together with translation history, translation practice and commentary constitute translation studies. The translation theory is mainly focused on the logical and theoretical research on translation; the history of translation is mainly on the evaluation and study of the history of translation; and the translation practice and comment on the practice of translation and the study of translation results. The three parts are closely related and interact. On the one hand, translation theory acquires material and data from the history of translation and translation practice and commentary, thus promoting the formation and development of its own system. On the other hand, it provides theoretical perspectives, methods and principles for translation history and translation practice; The research conclusions of the translation and translation reviews deal with the translation practice and history of translation theory, and describe the history of movement in the development of translation; translation commentary analyzes and analyzes phenomena such as translation texts, translation thoughts and translation schools, and on the one hand, it accepts translation theories. The guide, on the other hand, must "use history as a mirror."

Professor Cao Minglun made three explanations on the nature of translation theory. First of all, translation theory tends to study translation behavior and results from various perspectives such as literary studies, linguistics, semiotics, sociology, culturology, psychology anthropology, etc. However, the theory of these disciplines is not an integral part of translation theory. This is the so-called transdisciplinary study of translation theory. Second, translation is a common phenomenon as a social cultural phenomenon and other social cultural phenomena. In addition to recognizing this commonality, translation theories in particular must recognize translation as a unique social culture. The characteristics and laws of phenomena as well as their unique functions and effects. Third, translation practice will always occur and develop according to its own laws. Therefore, translation theory itself has a well-developed process.

\section{The Importance of Translation Theory in Foreign Language Translation Teaching}

Prof. Cao Minglun has studied this. According to his translation analysis theory, we can clearly see that he divides translation theory into ontology and comprehensive theory. Ontology is divided into pure translation theory and applied translation theory. It covers three aspects of theoretical knowledge. First, it uses the research results and theoretical methods of other disciplines to conduct theoretical research on translation issues. Second, it studies the translation phenomena subject to various social environments, cultural backgrounds, and ideologies. The study of translation as a means of social activity or cultural activity, etc. The author believes that ontology translation theory is the most important, necessary and indispensable part in the teaching of translation theory. The essence, principles, standards, operating procedures, methods, techniques, and translation history of translation must be taught to students. The cross-cultural and interdisciplinary studies on translation theory should be appropriate and appropriate to the students so that they can further study in the future.

Compared with traditional teaching methods, many scholars and professors advocate the process teaching method. The process pedagogy draws on the theoretical study of the translation process and reflects the actual process of translation and the main role of the translator. Therefore, it is conducive to developing students' translation skills. The process teaching method in translation teaching inspires students to understand the guiding principles, methods and operating procedures in the translation process; gives methodological and conceptual guidance prior to translation exercises; does not focus on commenting students' specific selections when discussing student translations. Sentences, but to explore the process of producing translations; the use of translation strategies has been vividly described and explained, allowing students to learn translation strategies 
faster; focusing on a certain type of problems in the translation process, reducing the energy dispersion; Reasons for appearance and reasons for good choices, understanding and understanding of the decision-making process in translation, not only focusing on the translation of words and phrases; flexibility in mastering the language forms and meanings of the translation, and faithfulness of the standard, fully allowing multiple expressions of translation, encouraging students' creativity By interpreting the process of translation production, the acceptance or denial of the translation is convincing; it avoids imposing the student's translation standards; it is particularly suitable for beginners who have been translating and has increased their awareness of translation issues from the beginning.

\section{Translation Theory and Its Application in Translation Teaching}

Related translation theory was developed on the basis of princip- ity related principles and related theories. This theory is a translation theory system formed by combining relevance theory with translation. It considers the process of translation of original texts to interpret the original texts. The communicative process mainly includes the two aspects of expressiveness and reasoning, that is, the translator expresses the original information and the original author's writing intention according to the original text, and combines their own cultural knowledge to think about the original text and optimize the reader's perspective with the original text. Correspondence; translators according to their own understanding of the original text, the original logical thinking and their own knowledge connected to each other, access to knowledge through reasoning, so as to convey to the target readers of the original text of the original text and the author's thoughts and feelings and writing intentions. In the perspective of associative translation theory, translation is not only the conversion between different languages, but also a dynamic reasoning process involving authors, translators, and readers, which makes the translation and translation readers reach the most appropriate Relevance is a key factor in the success of translation. Relevance translation theory is a new exposition of translation from the perspective of cognition. The proper application of the related translation theory in translation teaching will undoubtedly contribute to the long-term progress and development of translation teaching in China. According to the explanation of the related translation theory, the first step to be taken in translation is the translator's need to fully understand the meaning of the original text and the author's thoughts, feelings, and writing intentions. In the process of translation teaching, teachers should emphasize the understanding and analysis of the entire article, rather than excessive translations of sentences or phrases. They must combine different cultural backgrounds to reason about the intention of the wish text, and clarify the author's Writing intent. The related translation theory thinks that the second step of translation is the translator's understanding of the original meaning of the original text, which makes the reader of the translation and the original author resonate. Therefore, the teacher must pay attention to the cultivation of the students' comprehensive cultural knowledge in the process of translation. From the perspective of cultural background, students achieve the best relevance between the translation and the original meaning.

The use of discourse translation theory in the process of translation teaching is more accurate enough to guide students to grasp the discourse of an article, and to properly understand the original text's thoughts and feelings and writing intentions. Textual translation theory emphasizes that translators must fully understand the content of the original text, accurately grasp the structure of their articles and stylistic style, carefully savor the intentions and emotions of the original authors, and pay attention to the continuity of thinking between paragraphs and sentences. Grasp the level and significance of the article. In the traditional translation teaching, teachers usually guide the students' translation from the grammatical level. The problem of downgrading comes down to the weak grammar, which makes students pay too much attention to grammar and ignores discourse, which is not conducive to the cultivation of students' translation ability. Discourse translation theory helps guide teachers to break away from the traditional translation teaching model. Instead of stressing phrases or phrases too much, it starts from the whole chapter of the article and selects training materials of different styles and styles to cultivate students' Discourse analysis ability 
strengthens students' learning of different cultures, enabling students to fully understand the content of the original text and the true emotions and writing intentions the author wishes to express, in order to convey the most appropriate textual information for target readers.

\section{Conclusion}

In short, we must realize that translation teaching depends on the guidance of translation theory. The cultivation of translation talent depends on the exploration and development of translation teaching theory. Similarly, the establishment of a translation subject also depends on the study of translation theory. With the deepening and horizontal expansion of translation studies in both China and the West, the voice of establishing the discipline of translation has been rising. The importance of the basic work of translation theory has risen to a more prominent position. It has been taught in translation with translation practice. It plays an irreplaceable role and will eventually serve as a cornerstone for the establishment of a translation discipline.

\section{Acknowledgements}

The 13th Five-Year Social Science Research Project of the Department of Education of Jilin Province "Translation of Traditional Chinese Medicine in English Based on Information Technology"

"Study Practice and Research"

No.: JJKH2016 No. 10

\section{References}

[1] Xie Yanhong. The Application of Functional Translation Theory in College English Translation Teaching [J]. Journal of Luoyang Teachers College, 2010, 04:144-146.

[2] Ma Yuexi. Related Translation Theory and Its Application in Translation Teaching [J]. Xue Yuan, 2013, 27:66.

[3] Jiang Yong. The role of translation theory in translation teaching [J]. Journal of Kaifeng Institute of Education, 2014, 11:40-41.

[4] Zhong Xiaxia. Application of Interpretation Theory in College English Translation Teaching [J]. Journal of Hainan Radio and TV University, 2014, 04:143-146.

[5] Ren Huifang. Application of Text Translation Theory in Translation Teaching [J]. Reading and Writing (educational journal), 2014, 12:25-26. 